\title{
Role of Inpatient Pulmonary Rehabilitation Program in Acute Exacerbation of Chronic Obstructive Pulmonary Disease Patients: A Case Series
}

\author{
Rahmad, Djoko Witjaksono, Vivid Pretty A., Shabrina Narasati, Rakhmawati Diyana
}

\begin{abstract}
Chronic Obstructive Pulmonary Disease (COPD) defined as a chronic inflammatory lung disease that causes airflow obstruction and limitation of the lungs that primarily affects the airway and lung parenchyma. Pulmonary rehabilitation $(P R)$ in COPD have been shown improvement of respiratory capacity, exercise capacity, self-efficacy, quality of life, and reduce COPD-related hospital admission. The purpose of postural drainage, manual technique, and cough control is to drain the excessive sputum, while breathing control with pursed-lip breathing and breathing exercise with deep breathing exercise and chest expansion exercise are to improve the respiratory capacity and control. The purpose of this study was to investigate the feasibility and outcome of $P R$ in patients with Acute Exacerbation (AE) of COPD. This case report describes 4 patients with of $A E$ of $C O P D$ who received $P R$. The inpatient $P R$ program was 5 consecutive days twice daily of 3 times repetition cough control, 2 sets of 6 repetition pursed lip breathing, 2 sets of 6 repetition deep breathing exercise, postural drainage, and manual clapping. The postural drainage and the manual technique were given according to the chest $x$-ray result. The result of 5 days PR was: improvement of clean airway, SOB, incentive spirometer, chest expansion, and single breath count test in all subjects. Our findings demonstrate that early inpatient $P R$ in COPD AE is safe, significantly effective and beneficial by improving many aspects of respiratory function.
\end{abstract}

Keywords: Chronic Obstructive Pulmonary Disease, Pulmonary Rehabilitation.

\section{INTRODUCTION}

Chronic obstructive pulmonary disease (COPD) is one of the obstructive lung diseases characterized by a reduction in

Revised Manuscript Received on January 2, 2020.

* Correspondence Author

Rahmad, Department of Physical Medicine and Rehabilitation, Faculty of Medicine Brawijaya University, dr. Saiful Anwar General Hospital, Malang, Indonesia. Email: rahmadanwar1983@ub.ac.id

Djoko Witjaksono, Department of Physical Medicine and Rehabilitation, Faculty of Medicine Brawijaya University, dr. Saiful Anwar General Hospital, Malang, Indonesia.

Vivid Pretty A, Department of Physical Medicine and Rehabilitation, Faculty of Medicine Brawijaya University, dr. Saiful Anwar General Hospital, Malang, Indonesia.

Shabrina Narasati, Department of Physical Medicine and Rehabilitation, Faculty of Medicine Brawijaya University, dr. Saiful Anwar General Hospital, Malang, Indonesia. Email: snarasati@student.ub.ac.id

Rakhmawati Diyana*, Department of Physical Medicine and Rehabilitation, Faculty of Medicine Brawijaya University, dr. Saiful Anwar General Hospital, Malang, Indonesia. Email: rakhmawatid@student.ub.ac.id airflow and airflow limitation [1]. COPD is the most common type of lung disease and the third leading cause of chronic morbidity and mortality in the United States and worldwide, which is primarily attributable to its link with smoking [2,3].

Treatment of pulmonary disease involves a multipronged approach to achieve maximum benefit, which includes optimization of medal management, chest physical therapy, exercise training, oxygen therapy, and nutritional and psychosocial support [1]. Pulmonary rehabilitation (PR) for patients with COPD aims to improve disease management and exercise capacity $[1,4]$. In addition, pulmonary rehabilitation improves symptoms, respiratory capacity, physical and emotional participation, and quality of life of COPD patients [4].

Pulmonary rehabilitation is supported by the highest evidence level. A systematic review with meta-analyses by Rugbjerg et al [5] showed that significant improvement in short-term Health Related Quality of Life (HRQoL) following PR in mild symptoms COPD patients. Pulmonary rehabilitation is one of important components of the management of COPD and proven to be beneficial in improving the quality of life of COPD patients [6]. Another study by He et al [7] showed that PR is safe and feasible to implement as an early inpatient pulmonary rehabilitation program with acute exacerbation of COPD.

This case series was conducted to determine whether early pulmonary rehabilitation in COPD patient at inpatient setting following acute exacerbation is effective in improving pulmonary function capacities and quality of life.

\section{MATERIALS AND METHODS}

3 patients were recruited from July - September 2019. This case series used mixed methods of quantitative outcome assessment and also qualitative interviews. The inpatient pulmonary rehabilitation program consisting of 5 consecutive days supervised private sessions twice daily. The program included 3 times repetition cough control, 2 sets of 6 repetition pursed lip breathing, 2 sets of 6 repetition deep breathing exercise, postural drainage, and manual clapping. The postural drainage and the manual clapping were chosen according to the CXR.

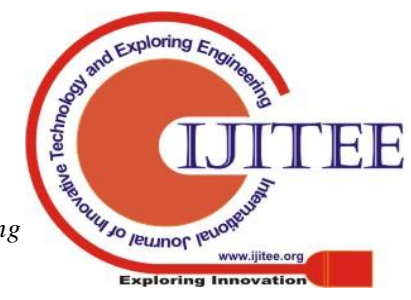




\section{Role of Inpatient Pulmonary Rehabilitation Program in Acute Exacerbation of Chronic Obstructive Pulmonary Disease Patients: A Case Series}

This case series was subsequently approved and registered at Ethical Committee of RSUD Dr. Saiful Anwar Malang.

Patients agreed to gave written informed consent to participate in the study at the first visit.

\section{CASE PRESENTATION}

\section{A. Case 1}

A 43-year-old male patient consulted by Pulmonology Ward at $1^{\text {st }}$ day of hospitalization with acute exacerbation COPD, inactive lung tuberculosis, and pneumonia. Patient complained of shortness of breath, high fever, and productive cough. At initial visit, the results of patient's assessment were as followed: pulmonary function capacities assessment showed inspiration capacity with incentive spirometry 675 cc/s; chest expansion 1,8 cm; Single-Breath Counting (SBC) 35; CXR results show infiltrate at apex on right and left upper lobe which indicate lung tuberculosis.

After 5 days of pulmonary rehabilitation, the examination results showed that there were clean airway, improvement of SOB, incentive spirometry was $1200 \mathrm{cc} / \mathrm{s}$, chest expansion $3,3 \mathrm{~cm}$, and SBC 30. The spirometry results on the day 5 showed force vital capacity (FVC) 65,1\%, forced expiratory volume in 1 second (FEV1) 30,3\%, and FEV1/FVC ratio $38,97 \%$ with conclusion mild restriction and moderate obstruction.

\section{B. Case 2}

A 47-year-old male patient consulted by Pulmonology Ward at $1^{\text {st }}$ day of hospitalization with acute exacerbation COPD and pneumonia. Patient complained of shortness of breath, high fever, and productive cough. At initial visit, the results of patient's assessment were as followed: pulmonary function capacities assessment showed inspiration capacity with incentive spirometry $900 \mathrm{cc} / \mathrm{s}$; chest expansion $1 \mathrm{~cm}$; Single-Breath Counting (SBC) 8; CXR results show infiltrate at lower right lobe which indicated COPD and pneumonia.

After 5 days of pulmonary rehabilitation, the examination results showed that there were clean airway, improvement of SOB, incentive spirometry was $1200 \mathrm{cc} / \mathrm{s}$, chest expansion 3 $\mathrm{cm}$, and SBC 21. The spirometry results on the day 5 showed FVC $72 \%$, FEV1 64,7\%, and FEV1/FVC ratio 74,64\% with conclusion mild restriction and mild obstruction.

\section{Case 3}

A 60 -year-old male patient consulted by Pulmonology Ward at $1^{\text {st }}$ day of hospitalization with acute exacerbation COPD and pneumonia. Patient complained of shortness of breath, high fever, and productive cough. At initial visit, the results of patient's assessment were as followed: pulmonary function capacities assessment showed inspiration capacity with incentive spirometry $600 \mathrm{cc} / \mathrm{s}$; chest expansion $1,5 \mathrm{~cm}$; Single-Breath Counting (SBC) 11; ADL by Barthel Index was 70; CXR results show COPD and pneumonia at right lower lobe.

After 5 days of pulmonary rehabilitation, the examination results showed that there were clean airway, improvement of $\mathrm{SOB}$, incentive spirometry was $900 \mathrm{cc} / \mathrm{s}$, chest expansion 2 $\mathrm{cm}$, SBC 20, and Barthel Index 100. The spirometry results on the day 5 showed FVC 47,5 \%, FEV1 $47.7 \%$, and FEV1/FVC ratio $100 \%$ with conclusion moderate restriction.

\section{TABLE-1: RESULT OF PULMONARY FUNCTION TEST AND QUALITY OF LIFE ASSESSMENT BEFORE AND AFTER INPATIENT PULMONARY REHABILITATION PROGRAM IN PATIENTS FOLLOWING ACUTE EXACERBATION OF COPD}

Indicator Patient 1 Patient 2 Patient 3

\section{Incentive Spirometer}

$\begin{array}{ll}\text { Incentive spirometer (H1) } & 675 \\ \text { Incentive spirometer (H2) } & 900 \\ \text { Incentive spirometer (H3) } & 1200 \\ \text { Incentive spirometer (H4) } & 1200 \\ \text { Incentive spirometer (H5) } & 1200 \\ \text { Chest Expansion } & \\ \text { Chest Expansion (H1) } & 1,8 \\ \text { Chest expansion (H5) } & 3, \\ \text { Single Breath Count (SBC) } & \\ \text { SBC (H1) } & 25 \\ \text { SBC (H5) } & 30 \\ \text { Insp (H1) } & 41 \\ \text { Exp (H1) } & 18 \\ \text { Pefr (H1) } & 140 \\ \text { Insp (H3) } & 41 \\ \text { Exp (H3) } & 20 \\ \text { Pefr (H3) } & 150\end{array}$

$\begin{array}{ll}900 & 600 \\ 1200 & 600 \\ 1200 & 600 \\ 1200 & 750 \\ 1200 & 900 \\ & \\ 1 & 1,5 \\ 3 & 2 \\ & \\ 8 & \\ 21 & 11 \\ 41 & 20 \\ 20 & 27 \\ 160 & 27 \\ 41 & 18 \\ 20 & 130 \\ 180 & 35 \\ & 20 \\ & 190\end{array}$




\begin{tabular}{llll}
\hline Insp (H5) & 41 & 41 & 41 \\
Exp (H5) & 20 & 20 & 20 \\
Pefr (H5) & 160 & & \\
Spirometry at Day 5 & & 72 & \\
FVC (\%) & 65,1 & 64,7 & 47,5 \\
FEV1 (\%) & 30,3 & 74,64 & 47,7 \\
FEV1/FVC (\%) & 38,97 & & 100 \\
& mild restriction and moderate obstruction. & mild restriction and mild obstruction. & restriction. \\
Result & & &
\end{tabular}

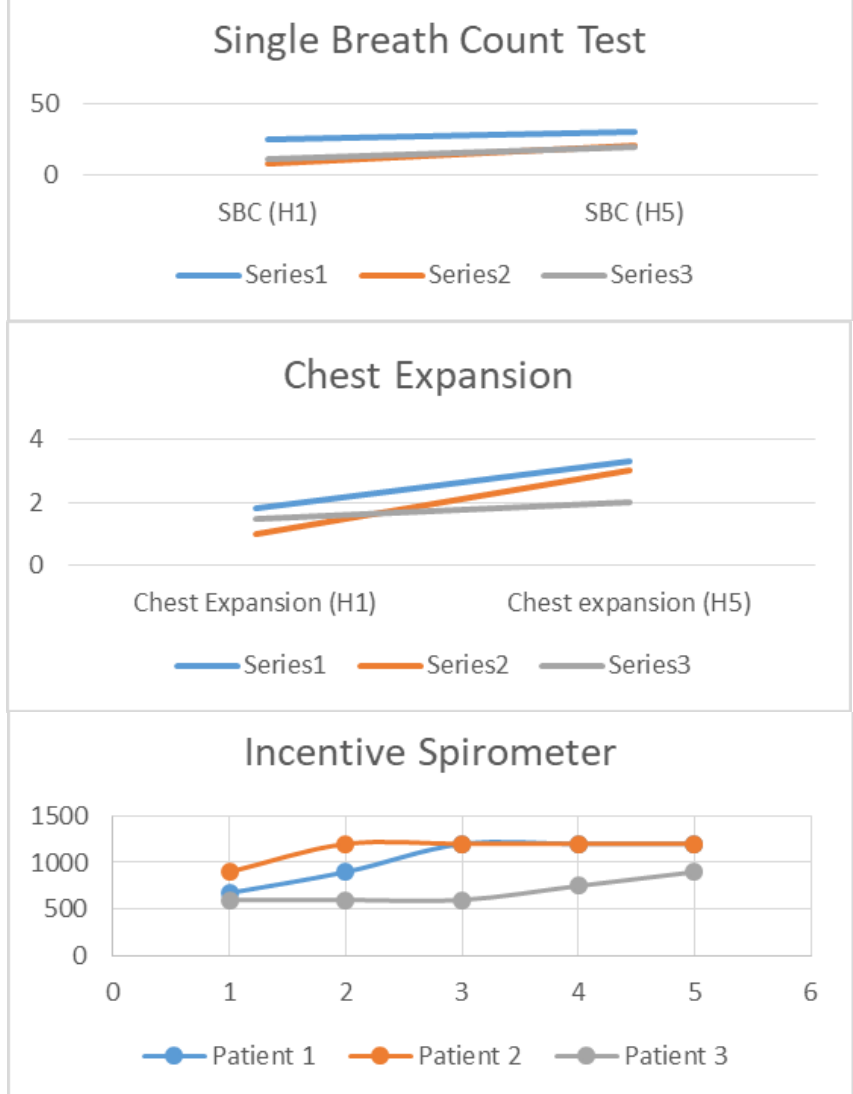

Fig. 1. Fig.1. Result of pulmonary function test including incentive spirometer, chest expansion, and single breath count test assessment before and after inpatient pulmonary rehabilitation program in patients following acute exacerbation of COPD.

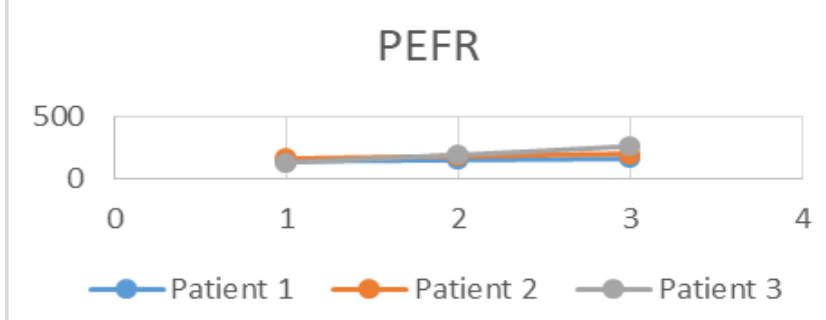

Fig. 2. Fig.2. Result Peak Expiratory Flow Rate (PEFR) assessment before and after inpatient pulmonary rehabilitation program in patients following acute exacerbation of COPD.

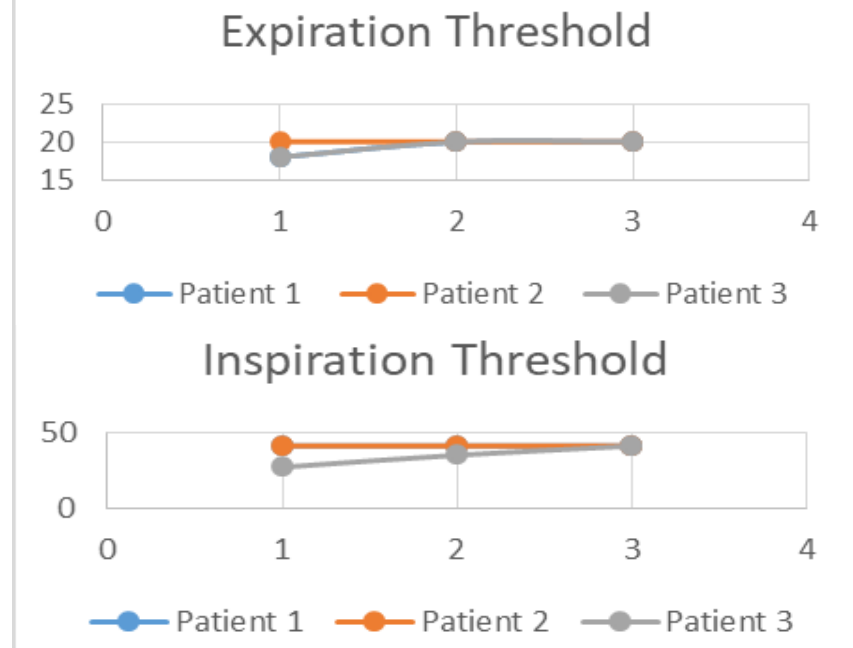

Fig. 3. Fig.3. Result inspiration and expiration threshold assessment before and after pulmonary rehabilitation program in a patient following acute exacerbation of COPD

\section{DISCUSSION}

The concepts of COPD rehabilitation has several complex and multimodal components consist of treatment optimization, education \& instruction, smoking cessation, exercise training \& breathing retraining, exacerbation management, and non-invasive ventilation [8]. Evidence based of pulmonary rehabilitation is supported by highest level evidence (A and B level). Pulmonary rehabilitation had A level evidence for improving quality of life, general physical performance ability, strength \& muscle mass, and reduction in resting and exertional dyspnea $[4,8]$. It is had B level evidence for reduction the hospital admissions and days spent in hospital also decrease in COPD associated anxiety and depression $[4,8]$.

There are several guideline in treating COPD. Our inpatient pulmonary rehabilitation programs are in line with the recommendation by The Global Initiative for Chronic Obstructive Lung Disease (GOLD) and American thoracic Society/European Respiratory Society (ATS/ERS) policy statement. The guidelines mention that COPD is a preventable and treatable lung disease that has several symptoms such as dyspnea, cough, sputum production, and also association with recurrent lower respiratory tract infection [4,9]. There is no difference of treatment management in the guidelines. The guidelines mention the treatments management of COPD are pharmacological with medication, smoking cessation, and also the

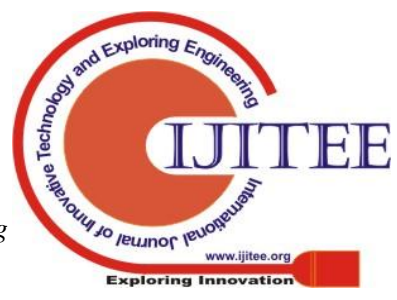




\section{Role of Inpatient Pulmonary Rehabilitation Program in Acute Exacerbation of Chronic Obstructive Pulmonary Disease Patients: A Case Series}

pulmonary rehabilitation [4,9].

Puhan et al [10] found that PR in acute exacerbation of COPD is clinically safe and beneficial in improving patient's exercise capacity and quality of life.

The goals of pulmonary rehabilitation are to reduced hospitalization and unscheduled healthcare visits, improve exercise capacity, reduce symptoms, improve muscle strength and endurance, quality of life, functional capacity, and also emotional function $[4,9]$. In this study, we found that pulmonary program can reduce the dyspnea, drain the excessive sputum, also improve the respiratory functions, which all above are improve the quality of life.

Eaton et al [11] found that early inpatient-outpatient pulmonary rehabilitation was feasible and safe in a randomized controlled study in 97 elderly patients hospitalized with COPD exacerbation. The pulmonary rehabilitation was associated with a non-significant trend in reducing COPD-related re-hospitalization and length of stays. There were no side effect finding of pulmonary rehabilitation in acute exacerbation of COPD, so pulmonary rehabilitation was estimated safe.

Study of Jette et al [12] in 64-year-old woman taking part in pulmonary rehabilitation program in outpatient care after 3 COPD exacerbations over 4-months period has similar results with this study. There were clinically significant improvements shown in patient's quality of life as well as exercise capacity.

Although there were improvement of symptoms and respiratory functions in acute exacerbation COPD patients with our pulmonary rehabilitation program, our study has several limitation. First, this study has limited patient and there is no follow-up data of post hospitalization. Second, this study use simple measurements as the indicators of respiratory function, but still, there are more complete measurement that can be used for respiratory function evaluation. However, this also a benefit that this method only need simple measurements that can be done at all health facilities. Further larger study is needed to evaluate the optimal effectiveness of this method, including long-term follow-up data and more complete measurement.

\section{CONCLUSION}

Early inpatient pulmonary rehabilitation programs give benefit by reducing symptoms and improving many aspects of respiratory function in acute exacerbation of COPD hospitalized patient. Pulmonary rehabilitation program in this study aims to improve airway cleaning, control breathing and cough, and strengthen respiratory muscles that lead to improve patient's respiratory function capacities.

\section{REFERENCES}

1. Cifu D, (2016). Braddom's Physical Medicine And Rehabilitation, Elsevier, Philadelphia.

2. Centers for Disease Control and Prevention: Data and statistics - chronic obstructive pulmonary disease (COPD), (2014) Centers for Disease Control and Prevention. Available at: <http:// www.cdc.gov/copd/data.htm>. Accessed December 20, 2019

3. Lozano R, Naghavi M, Foreman K, et al., (2010) Global and regional mortal- ity from 235 causes of death for 20 age groups in 1990 and 2010: a systematic analysis for the Global Burden of Disease Study, Lancet 380(9859):2095-2128, 2012.
4. GOLD. (2019). Pocket Guide to COPD Diagnosis, Management, and Prevention: A Guide for Healthcare Professional, 2019 Edition. Global Initiative for Chronic Obstructive Lung Disease, Inc

5. Effectiveness of pulmonary rehabilitation in COPD with mild symptoms: a systematic review with meta-analyses. International Journal of COPD 2015:10 791-801

6. Jige Dong, Zhanjun $\mathrm{Li}^{\mathrm{b}}$, Lihua Luo ${ }^{\mathrm{a}}$, Hongzhi Xie., (2019) Efficacy of pulmonary rehabilitation in improving the quality of life for patients with chronic obstructive pulmonary disease: evidence based on nineteen randomized controlled trials - a systematic review and meta-analysis. Int J Surg.

7. He M, Yu S, Wang L, Lv H, Qiu Z, (2015). Efficiency and safety of pulmonary rehabilitation in acute exacerbation of chronic obstructive pulmonary disease. Med Sci Monit, 21:806-812.

8. Gloeckl, R., Schneeberger, T., Jarosch, I. and Kenn, K., (2018). Pulmonary rehabilitation and exercise training in chronic obstructive pulmonary disease. Deutsches Ärzteblatt International, 115(8), p.117.

9. Rochester, C.L., Vogiatzis, I., Holland, A.E., Lareau, S.C., Marciniuk, D.D., Puhan, M.A., Spruit, M.A., Masefield, S., Casaburi, R., Clini, E.M and Crouch, R., (2015). An official American Thoracic Society/European Respiratory Society policy statement: Enhancing Implementation, Use, and Delivery of Pulmonary Rehabilitation. American journal of respiratory and critical care medicine, 192(11), pp.1373-1386.

10. Puhan MA, Scharplatz M, Troosters T, Walters EH, Steurer J. (2009) Pulmonary rehabilitation following exacerbations of chronic obstructive pulmonary disease. Cochrane Database Syst Rev. (1):CD005305.

11. Eaton T, Young P, Ferguson W, Moodie L, Zeng I, O'kane F, et al. (2009). Does early pulmonary rehabilitation reduce acute health-care utilization in COPD patients admitted with an exacerbation? A randomized controlled study. Respirology. 14, 230-238.

12. Jette DU, Bourgeois MC, Buchbinder R. (2010). Pulmonary rehabilitation following acute exacerbation of chronic obstructive pulmonary disease. Physical Therapy. 90(1):9-12.

\section{AUTHORS PROFILE}

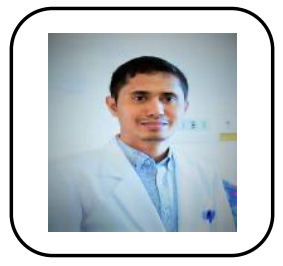

Rahmad, graduate as an MD from Faculty of Medicine Brawijaya University and finished his residency from Department of Physical Medicine and Rehabilitation, Faculty of Medicine Airlangga University. Now is a lecturer at Department of Physical Medicine and Rehabilitation, Faculty of Medicine Brawijaya University.

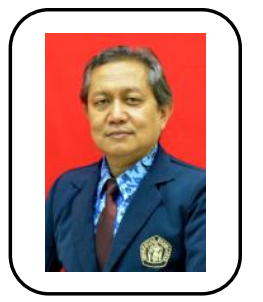

Djoko Witjaksono, graduate as an MD from Faculty of Medicine Airlangga University and finished his residency from Department of Physical Medicine and Rehabilitation, Faculty of Medicine Airlangga University. Now is a lecturer at Department of Physical Medicine and Rehabilitation, Faculty of Medicine Brawijaya University.

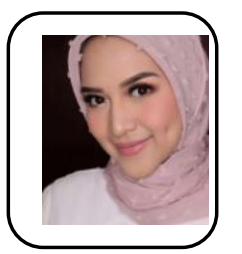

Shabrina Narasati, graduate as an MD from Faculty of Medicine Indonesia University and now is a resident of Department of Physical Medicine and Rehabilitation, Faculty of Medicine Brawijaya University.

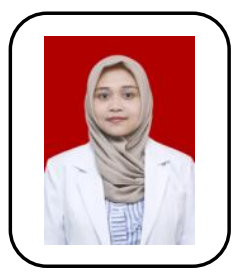

Rakhmawati Diyana*, graduate as an MD from Faculty of Medicine Brawijaya University and now is a resident of Department of Physical Medicine and Rehabilitation, Faculty of Medicine Brawijaya University.

Vivid Pretty A, graduate as an MD from Faculty of Medicine Airlangga University and finished his residency from Department of Physical Medicine and Rehabilitation, Faculty of Medicine Airlangga University. Now is a lecturer at Department of Physical Medicine and Rehabilitation, Faculty of Medicine Brawijaya University. 